\title{
Tumor ovárico bilateral de bajo potencial de malignidad en mujer joven y nulípara
}

\author{
Gilberto Martínez Morales*, Gonzalo Angel**
}

\begin{abstract}
RESUMEN: Se presenta el caso de una paciente de 20 años de edad quien ingresó con cuadro clínico de tumor de ovario derecho sospechosos de malignidad de 17 x 15 x $8 \mathrm{cms}$. Los estudios paraclínicos de extensión fueron negativos y el CA-125 fue de 196 U/ml. A la exploración quirúrgica se confirmó tumor limitado al ovario derecho, la biopsia por congelación reportó tumor Mulleriano Pseudomucinoso de Bajo Potencial de Malignidad por lo cual se realizó cirugía conservadora incluyendo cuña del ovario contralateral donde se observaba un pequeño quiste de $\mathrm{lcm}$ de aspecto benigno, lavado peritoneal, omentectomía infracólica, biopsia ganglionar paraaórtica y apendicectomía. La patología definitiva reportó Tumor Quístico^Papilar Seromucinoso de Bajo Potencial de malignidad del Ovario Derecho y cuña del ovario izquierdo con Tumor Quístico Papilar Seromucinoso de Bajo Potencial de malignidad con bordes de sección libres de tumor, los lavados peritoneales, epiplón, apéndice y glanglios retroperitoneales libres de tumor. Estos resultados permitieron dejarla en observación con controles clínicos períódicos, conducta que puede ser tomada en casos similares en pacientes jóvenes sin hijos y con deseos de reproducción. Se revisa la literatura y se analiza el tema.
\end{abstract}

PALABRAS CLAVES: Ovario, tumor, potencial de malignidad, bilateral, jóvenes, nulíparas

SUMMARY: A 20 years old patient who was admited with a right ovary tumor case suspicious of malignancy is discussed. The paraclinic extension studies were negatives and the $\mathrm{CA}-125$ was $196 \mathrm{U} / \mathrm{ml}$. At laparotomy it was confirm a right ovary limited tumor, the congelation biopsy reported a Low Malinan Potential Pseudomucinosus Mullerian Tumor; for this razon a conservative surgery was done, including a contralateral wedge biopsy where a $1 \mathrm{~cm}$ cistic lesion of benign aspect was observed, peritoneal citology washing, infracolic omentectomy, paraaortic nodes biopsy and apendectomy. The definitive anatomopathological report a Low Malignant Potential Papilar Seromucinosus Cistic Tumor in the rigth ovary and in the wedge biopsy of the left ovary with free tumor margens; the peritoneal washings citology, the omento, apendice and retroperitoneal nodes were negatives for extension and metastatic disease. This results allowed maintein the patient in observation with clinical periodic controls, conduct that must be taken in similar cases in young patients without children and reproduction whishes. Literature is revised and the teme is being analized.

KEY WORDS: Ovary, tumor, low malignant potential, bilateral, young, nuliparous.

Paciente de 20 años, soltera, empleada de oficios domésticos, sin antecedentes patológicos ni quirúrgicos, nuligestante fue remitida al Instituto Nacional de Cancerología con cuadro de 4 meses de evolución de crecimiento abdominal, sensación de masa y dolor pélvico; traía TAC abdomino-pélvico donde se observó gran masa de predominio quístico con presencia de paredes irregulares y lesiones papilares en su interior, con diámetros de $17 \times 15 \times 8 \mathrm{~cm}$. dependiendo de ovario derecho y con efecto compresivo sobre ureter derecho. Sus períodos menstruales eran normales y su última menstruación fue tres días antes de la consulta inicial.

En el examen físico se encontró paciente en aceptable estado general, sin adenopatías periféricas, mamas clínicamente normales, marcada distensión abdominal por la presencia de masa de consistencia quística, lisa y muy móvil que ocupaba casi todo el abdomen. El examen ginecológico evidenció vagina y cuello sanos, escaso sangrado menstrual e histerometría de $8 \mathrm{cms}$. Al tacto bimanual presencia de masa palpada en abdomen con

\footnotetext{
* Jefe de Ginecología, Instituto Nacional de Cancerología. ** Instructor de Ginecología, Instituto Nacional de Ginecología
}

igual consistencia y que rechazaba útero hacia atrás. Se tomó biopsia de endometrio. Los estudios de laboratorio demostraron hemoglobina $14.7 \mathrm{gm} / \mathrm{dl}$, Rx de tórax normal, parcial de orina normal, citología vagina PAP II, biopsia de endometrio: endometrio proliferativo descamando. Marcadores tumorales séricos CEA $1.4 \mathrm{ng} /$ ml., HCG o mud/ml, CA 125196 U/ml (normal menor de $35 \mathrm{U} / \mathrm{ml}$ ).

Se realizó laparotomía exploradora encontrando líquido peritoneal $60 \mathrm{cc}$ de aspecto citrino, las serosas peritoneales, cúpulas diafragmáticas, palpación de vísceras y cadenas ganglionares fueron de aspecto normal. Masa dependiente del ovario derecho de $20 \mathrm{~cm}$. de diámetro de superficie tensa, lisa, blanco nacarada, translúcida y libre de adherencias. El ovario izquierdo de aspecto folicular de tamaño normal con una pequeña lesión de $1 \mathrm{~cm}$. de aspecto quístico cristalino que protruía de la superficie. Se practicó toma de muestras así: líquido peritoneal, lavado de goteras parietocólicas, citología de cúpulas diafragmáticas, se realizó salpingoooforectomía derecha, biopsia por congelación de la misma que se informó como Tumor Mulleriano Seromucinoso de bajo potencial de malignidad, por lo que se realizó cirugía conservadora y clasificatoria que incluyó biopsia 
cuneiforme del ovario contralateral, omentectomía infracólica, biopsia ganglionar paraaórtica, paracavos y apendicectomía.

La patología definitiva informó tumor quístico papilar seromucinoso de bajo potencial de malignidad en ovario derecho, cuña de ovario izquierdo con tumor quístico papìlar seromucinoso de bajo potencial de malignidad con bordes de resección libres de tumor, quistes luteínicos y cuerpos blancos. Los ganglios precavos y paraaórticos negativos para tumor, epiplón y apéndice cecal normales. Líquido peritoneal, lavado de goteras parietocólicas y citología de cúpulas diafragmáticas negativas. Evolución post operatoria satisfactoria y controles posteriores normales.

\section{Discusión}

El cáncer ovárico epitelial es el subtipo histológico más común y representa el $90 \%$ en mujeres adultas. La mayoría de los casos ocurre en mujeres post-menopáusicas con edad promedio de 61 años (1.3). Es rara en niños y adolescentes, la mayoría de los tumores ováricos en esta edad se encuentran en estadío, en el momento del diagnóstico y el manejo conservador con preservación de la fertilidad es posible. En general las neoplasias en esta grupo son de tipo germinal (2). Sin embargo los de tipo epitelial se pueden presentar rara vez, especialmente tumores de bajo potencial de malignidad.

El cáncer ovárico epitelial ocupa el primer lugar como causa de muerte por malignidad ginecológica en Estados Unidos con una supervivencia global del $37 \%$ en relación con el alto porcentaje de pacientes que se presentan en estadío avanzado. En Colombia es la segunda causa de mortalidad. El carcinoma epitelial de ovario es raro por debajo de los 40 años de edad y se incrementa su incidencia al avanzar la edad con un pico de incidencia del 54/100.000 en mujeres de 75 a 79 años. $(1,3,8)$.

Los datos del (SEER) programa de vigilancia, epidemiología y resultado final, del Instituto Nacional de Cáncer indican una significativa mejoría en la sobrevida en mujeres más jóvenes. Parte de esta diferencia en la sobrevida está relacionada con el hecho de que mujeres de mayor edad se presentan con enfermedad más avanzada al momento del diagnóstico, aproximadamente $75 \%$ de las mujeres mayores de 65 años lo hacen en estadíos III y IV comparado con sólo el $47 \%$ en pacientes menores de 45 años (3).

Esto también refleja la más alta incidencia de tumores de bajo potencial de malignidad en mujeres más jóvenes y el hecho de que por lo menos la mitad de ellas se encuentra en estadío I y las dos terceras partes en estadíos I y II (enfermedad que no rebasa la pelvis). (4).

Gronroos informó la frecuencia de tumores de bajo potencial de malignidad y cáncer ovárico verdaderos, relacionados con la edad y encontró que entre el $50 \%$ y el $80 \%$ de los tumores de bajo potencial de malignidad se presentan en mujeres por debajo de los 49 años de edad (5).

Frecuencia de los tumores Borderline versus verdaderos Carcinoma Ovárico con relación a la edad.

\begin{tabular}{|ccccc|}
\hline Edad & BL & Carcinoma & Total & $\%$ BL \\
\hline $20-29$ & 5 & 1 & 6 & $(83)$ \\
$30-39$ & 10 & 5 & 15 & $(67)$ \\
$40-49$ & 17 & 18 & 35 & $(49)$ \\
$50-59$ & 19 & 35 & 54 & $(35)$ \\
$60-69$ & 9 & 28 & 37 & $(24)$ \\
$>70$ & 1 & 4 & 5 & $(20)$ \\
\hline
\end{tabular}

Tomado de Gronroos, Lauren, Lehto y Rauraneo, 1969.

La tasa global de sobrevida para tumores de bajo potencial de malignidad es mayor del $90 \%$ a 10 años (6), por lo que Massad y colaboradores lo exponen en términos de incidencia de muerte por estadío de enfermedad así: E-I 1.9\%; E II 5.9\%; E III y IV 21\%. De donde se corrobora que el estadío sigue siendo el factor pronóstico más importante para tumores de bajo potencial de malignidad con una diferencia de 10 veces entre pacientes con enfermedad estadío I y Estadíos III y IV. $(7,8)$.

La mayoría de los tumores de bajo potencial de malignidad son serosos y muscinosos pero puede presentarse en cualquiera de los tumores epiteliales. Los criterios para definirlos están claramente establecidos y fueron unificados y aprobados desde 1973 por la OMS, así: Estratificación de las células epiteliales, desprendimiento aparente de penachos celulares de su sitio de origen, actividad mitótica y anomalías nucleares intermedias entre aquellas claramente benignas y francamente malignas, ausencia de invasión del estrógeno. (9).

El tratamiento convencional para pacientes con tumores de bajo potencial de malignidad es la histerectomía abdominal total con salpingo ooforectomía bilateral en pacientes perimenopáusicas o con paridad deseada completa; si la enfermedad está avanzada deben disminuirse volúmenes tumorales según esté indicado. La clasificación debe ser exhaustiva especialmente para quienes tienen enfermedad confinada al ovario.

La menor edad de estas mujeres y el diagnóstico en etapas más tempranas de los tumores de bajo potencial de malignidad, convierte estas pacientes en candidatas ideales para cirugía conservadora, especialmente cuando preocupa la fertilidad futura. Varios autores han publicado estudios acerca de la evolución del tratamiento conservador.

Lim Tan y colaboradores informaron 35 casos tratados mediante cistectomía ovárica unilateral o bilateral, con persistencia o recidiva del $6 \%$ en estadío I. En ovario ipsilateral y contralateral en un paciente $(3 \%)$. En el contralateral en un paciente (3\%), para una tasa global del $12 \%$. Se determinó que dos factores eran predictivos de recidiva: la multifocalidad de la entidad y la afección de los bordes de resección. 8 de estas pacientes tratadas conservadoramente lograron embarazo. A pesar de las recidivas todas las mujeres se mantienen vivas y libres de enfermedad en un tiempo promedio de vigilancia de 6.5 años, incluyendo las que requirieron nueva cirugía. (10).

Tazelaar y colaboradores encontraron recidivas en 3 de 20 pacientes $(15 \%)$ en quienes se efectuó cirugía conservadora en comparación con 2 de 29 pacientes ( $7 \%$ ) en quienes se realizó histerectomía abdominal total más 
salpingo ooforectomía bilateral con una vigilancia promedio de 89 meses, todas las pacientes se encontraban vivas, libres de enfermedad incluyendo quienes presentaron recidiva. (11)

Kliman y colaboradores informaron 56 mujeres con tumores de bajo potencial de malignidad en estadio I, 19 de las cuales fueron objeto de ooforectomía. La única recidiva ocurrió en una paciente que fue sometida a histerectomía abdominal total mas salpingo ooforectomía unilateral 3 años después en el ovario residual. (12)

Rice y colaboradores no observaron recidivas en 30 casos de enfermedad en etapa I tratados conservadoramente incluyendo 10 pacientes a quienes solo se les practicó cistectomía (13).

Estos y otros datos indicarían que pacientes con enfermedad en estadíos tempranos deseosas de procreación futura, pueden tratarse de manera conservadora, haciendo énfasis en una exhaustiva clasificación quirúrgica ya que también debe recordarse que entre el 15 y $20 \%$ de las pacientes se presentan con enfermedad extraovárica y un número importante de ellas puede hacerse únicamente manifiesto por metástasis gnaglionar (pélvica y para - aórtica). (14-15). La apendicectomía se justifica especialmente en tumores mucinosos ya que se ha vinculado la presencia de tumor apendicular primario sincrónico. (16). La biopsia de ovario contralateral aunque sistemática también es controvertida. La bilateralidad se presenta en el $38 \%$ de los serosos y en el $8 \%$ de los mucinosos. (7). En el caso que presentamos se apreció intraoperatoriamente ovario izquierdo de aspecto nor- mal con pequeña formación quística de aspecto inofensiva y que sorpresivamente la patología lo confirmó como tumor seromucinoso papilar de bajo potencial de malignidad, por fortuna para la paciente con márgenes libres de tumor. En este caso se presentó bilateralidad.

El seguimiento se hace en igual forma que para pacientes con verdaderos carcinomas ováricos: examen físico, ecografía abdominopélvica y CA-125.

En términos generales los tumores de bajo potencial de malignidad cursan con bajos títulos de CA-125, dentro de lo normal o ligeramente elevados y se negativisan rápidamente luego de la cirugía en promedio 1 ó 2 semanas.

En resumen acerca de los tumores ováricos de bajo potencial de malignidad puede ser dicho que representan el $10 \mathrm{al} 15 \%$ de todos los tumores epiteliales ováricos, que la edad promedio de aparición está entre los 43 y 53 años, el $80 \%$ de los casos se encuentran en estadío I, la sobrevida global a 10 años libre de enfermedad es de $95 \%$ y que aún tumores avanzados tienen un curso indolente y solo un pequeño porcentaje de los casos es fatal. $(17,8,18)$.

Finalmente recordar que el manejo juicioso conservador no solo permite la posibilidad de procreación futura, sino que con un bajo riesgo se puede conservar la función hormonal ovárica en una mujer joven que de lo contrario con una cirugía radical "salvadora de vida" (para una tasa baja de mortalidad en estadío inicial) sí garantiza una enfermedad definitiva por castración además de la infertilidad.

\section{BIBLIOGRAFIA}

1. National Cancer Institute, Division of cancer prevention and control: annual cancer statistics review. Bethesda, NIH Publication No. 872789, 1986.

2. Breen, J. L., and Maxson, W. S. Gynecol. 154, 639-647.

3. Yancik, R., Ries, L. G., and Yates, J. W. Ovarian Cancer In The Elderly: An Analysis of Surveillance, Epidemiology And End Results Program Date. Am. J. Obstet. Gynecol. 1986; 154: 639-697.

4. Annual Report Gynecologic Cancer FIGO 1988 Petterson F. (Ed) Panorama Press, Stockholm, Vol. 20.

5. Gronroos M, Lauren P, Lehto J, Rauramo L. 1969 Ovarian Cancer And Treatment. Ann. Chir. Gynaecol. Fenn. 58: 83.

6. Aure J.C., Hoeg K, Kolstad P: Clinical And Histologic Studies of Ovarian Carcinoma: Long Term Follow-Up of 990 Cases, Obstet Gynecol. 1971; 37: 1 .

7. Massad L.S.Jr., Hunter V.J., SzpakC.A., Et al: Epithelial Ovarian Tumors of Low Malignant Potencial. Obstet Gynecol. 1991; 78: 1027

8. Richard R. Barakat, Borderline Tumor of the ovary, Obstetrics And Gynecology Clinics of North America. 1994.

9. Serov S.F., Scully R.E., Sobin L. H.: International histological, Classification and Staging of Tumors. No. 9 Histologic Typing of Ovarian Tumors. Geneva, World Health Organization, 1973.
10. Lim Tan S.R. , Cjigas H.E., Scully R,.E.. Ovarian Cystectomy For Serous Borderline Tumors. Obstet Gynecol. 1985; 66: 417.

11. Tazelaar H.D., Bostwick D.G., Ballon S.C., Et al: Conservative treatment of Borderline ovarian Tumors. Obstet Gynecol. 1985; 66: 417.

12. Kliman L., Rome R.M., Fortune D.W.: Low Malignant Potencial Tumors of the Ovary: A Study of 76 cases. Obstet Gynecol. 1986; 68: 338.

13. Rice W.R., Berkowitz R.S., Mark S., Et al: Epithelial Ovarian Tumor of Borderline Malignancy. Gynecol Oncol. 1990; 39: 195.

14. Sutton G. P., Bundy B.N. Omura G.A., Et al: Stage III Ovarian Tumors of Low Malignant Potencial. (A Gynecologic Oncology Group Study) Gynecol Oncol. 1991; 41: 230.

15. Leake J.F., Raden J.S., Woodrwff J.D., Et al: Retroperitoneal Lynphatic Involuewent Ovarian Tumores of Low Malignant Potencial: Gynecol Oncol 42: 124, 1991.

16. Rutger J.L., Scully R.E.: Ovarian Mullerian Mucinous Papillary Cistadenomas of Borderline Malignancy. Cancer. 1988; 61: 340.

17. Philip J. Disaia, William T. Creastwan: Clinical Gynecologic Oncology. Fourth Edition: 1993; 320-346.

18. Malcolw Copleson: Gynecologic Oncology Fundamental Principles and Clinical Practics, Second Edition 836, 1992. 